\title{
Therapiestandards und Perspektiven beim nichtkleinzelligen Lungenkarzinom im Stadium IV
}

\author{
Martin Reck ${ }^{\mathrm{a} *}$ Rafael Rosell ${ }^{\mathrm{b} *}$ Markus Tiemann ${ }^{\mathrm{c} *}$ \\ ${ }^{a}$ Onkologischer Schwerpunkt, Krankenhaus Großhansdorf, Deutschland \\ ${ }^{\text {b } C a t a l a n ~ I n s t i t u t e ~ o f ~ O n c o l o g y, ~ H o s p i t a l ~ G e r m a n ~ T r i ́ a s ~ i ~ P u j o l, ~ B a r c e l o n a, ~ S p a n i e n ~}$ \\ ${ }^{\circ}$ Institut für Hämatopathologie, Hamburg, Deutschland
}

\section{Therapeutischer Algorithmus im Stadium IV}

Die Prognose beim fortgeschrittenen nichtkleinzelligen Lungenkarzinom (NSCLC) konnte in letzter Zeit deutlich verbessert werden. Zurückzuführen ist dies nicht nur auf die Entwicklung neuer Arzneimittel und die zunehmende Akzeptanz einer Erhaltungstherapie, sondern auch auf die stärkere Selektionierung der von einer bestimmten medikamentösen Therapie voraussichtlich profitierenden Patienten. Während bei der Therapieentscheidung früher meist nur das Tumorstadium und der Allgemeinzustand berücksichtigt wurden, werden als Selektionsfaktoren inzwischen auch die Tumorhistologie, die Eignung für eine Bevacizumab-Therapie, das Ansprechen auf die Induktionstherapie und das genetische Profil des Tumors genutzt.

Die prädiktive Bedeutung der Histologie für ein Ansprechen auf Pemetrexed zeigte unter anderem die Analyse von 3 randomisierten Phase-III-Studien, in denen Patienten mit nichtsquamösem NSCLC einen signifikanten Überlebensvorteil im Vergleich zu Patienten mit Plattenepithel-Histologie hatten [1]. Zurückzuführen ist dies möglicherweise auf die bei Patienten mit Adenokarzinom niedrigere Expression der Thymidylat-Synthase, einem wichtigen Target des Antifolats [2].

Patienten, die für eine Erstlinien-Therapie mit Bevacizumab geeignet sind, profitieren von der Zugabe des VEGFAntikörpers (VEGF = vaskulärer endothelialer Wachstumsfaktor) zu einer platinhaltigen Dublette mit einer signifikanten Verbesserung der Ansprechrate und Prognose. In einer Metaanalyse von 6 klinischen Studien verringerte Bevacizumab statistisch signifikant sowohl das Mortalitätsrisiko (Hazard Ratio (HR) 0,90; $\mathrm{p}=0,03$ ) als auch das Progressionsrisiko (HR 0,72; $<<0,001)$ [3].

*Alle Autoren haben zu gleichen Teilen zu diesem Artikel beigetragen. Auf Vorschlag der Autoren wurde der von Rafael Rosell verantwortete Abschnitt in englischer Sprache belassen und nicht ins Deutsche übersetzt.
Patienten mit Ansprechen (Krankheitsstabilisierung oder Remission) auf eine Erstlinien-Therapie kommen für eine Erhaltungstherapie infrage, bei der entweder eine neue Substanz verabreicht wird (Switch Maintenance) oder die Behandlung mit 1 bzw. 2 der zuvor in der Induktionstherapie eingesetzten Substanzen fortgeführt wird (Continuous Maintenance). Für beide Therapieansätze zeigen die bisher verfügbaren Daten randomisierter Studien eine signifikant verbesserte Wirksamkeit im Vergleich zu Placebo bzw. zur Kontrollmedikation.

Durch Mutationsanalysen können Patienten mit aktivierender Mutation des epidermalen Wachstumsfaktorrezeptors (EGFR) selektioniert werden, die in randomisierten Studien bei einer Erstlinien-Therapie mit Erlotinib oder Gefitinib Ansprechraten von etwa 60-80\% erreichten. Das Progressionsrisiko konnte im Vergleich zu einer Standard-Chemotherapie um etwa 50-80\% verringert werden.

Der unter Einschluss der neuen Stratifizierungsfaktoren entwickelte Therapiealgorithmus (Abb. 1) spiegelt die aktuelle Therapiesituation wider [4], erfordert aber auch zukünftig kontinuierlich Anpassungen an die Entwicklungen in der personalisierten Medizin.

\section{Entwicklungen im Stadium IV}

Während bei der zielgerichteten Therapie des fortgeschrittenen NSCLC inzwischen 2 zur Patientenselektion geeignete molekulare Marker (aktivierende EGFR-Mutation, EML4ALK-Translokation) bekannt sind, konnten bei der Chemotherapie des NSCLC im Stadium IV bisher keine prädiktiven Biomarker identifiziert werden. Bei einer auf Basis der ERCC1-Expression personalisierten Chemotherapie wurde in der GILT-Studie im experimentellen Arm zwar eine Verbesserung der objektiven Ansprechrate (primärer Endpunkt) gesehen, das Gesamtüberleben war im Vergleich zum Kontrollarm ohne ERCC1-basierte Chemotherapie aber nicht signifikant verbessert [5]. Die Wirksamkeit einer personalisierten

\section{KARGER}

Fax +497614520714

Information@Karger.de

www.karger.com
(C) 2012 S. Karger GmbH, Freiburg

0378-584X/12/3515-0010\$38.00/0 


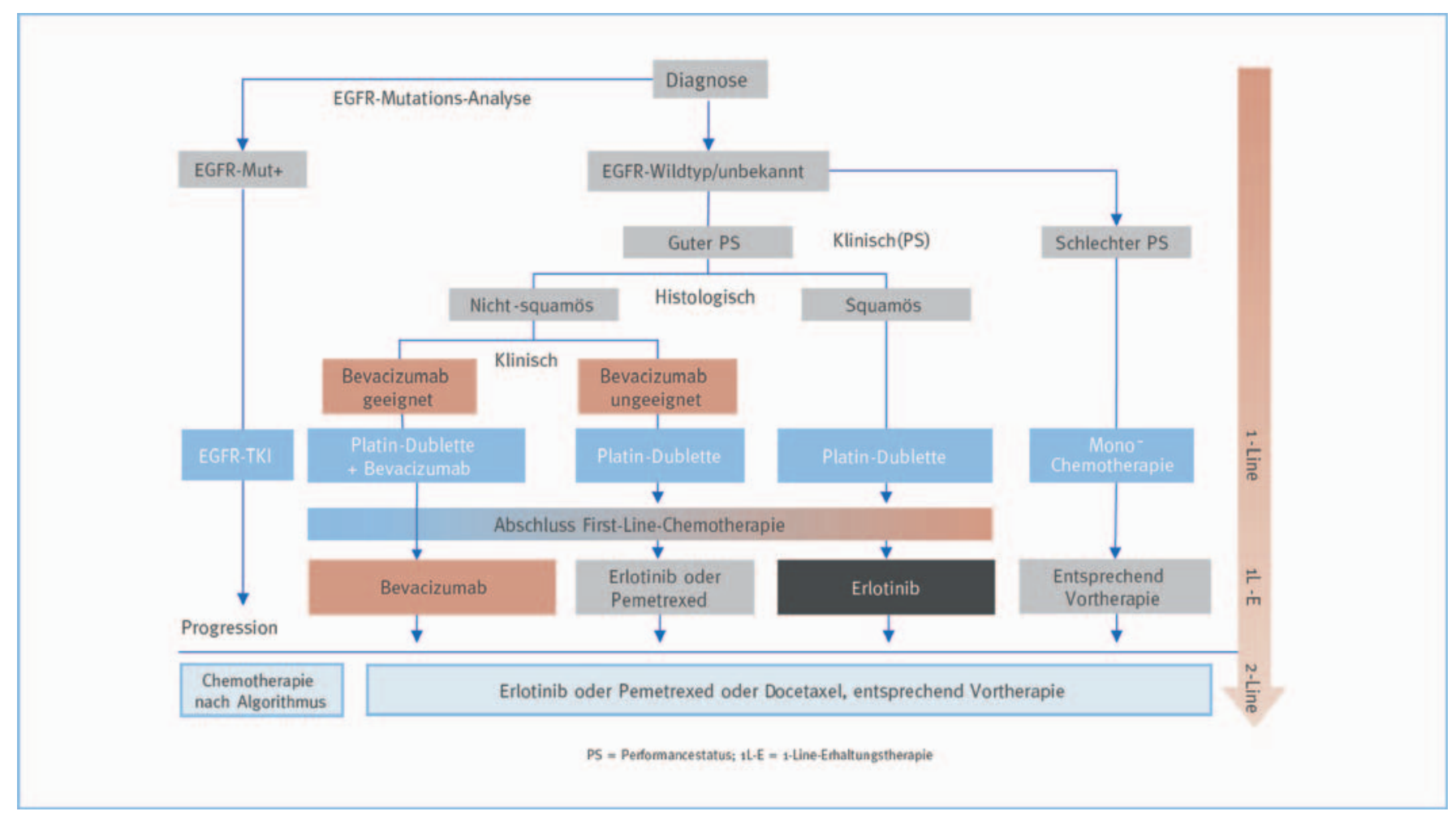

Abb. 1. Therapiealgorithmus bei Patienten mit fortgeschrittenem NSCLC (modifiziert nach [4]).

Chemotherapie auf Grundlage des RAP80- und BRCA1Spiegels wird zurzeit im weltweiten BREC-Programm (BREC = BRCA1 RAP80 Expression Customization) untersucht.

\section{Ausblick}

Je mehr molekulare Marker identifiziert werden, desto wichtiger wird die Etablierung eines diagnostischen Algorithmus, der unter Berücksichtigung der Exklusivität einzelner molekularer Veränderungen auch bei sehr kleinen Tumorproben valide Aussagen zu den therapierbaren molekularen Veränderungen ermöglicht. Bei Patienten mit Progression wird zu klären sein, ob die Folgetherapie auf Basis der ersten Tumorprobe erfolgen kann oder möglicherweise eine Rebiopsie erforderlich ist.

\section{Variety of EGFR Mutations and Clinical Impact}

In European patients with newly diagnosed, advanced NSCLC and proven activating EGFR mutation, erlotinib reduced the risk of progression compared to standard chemotherapy by a statistically significant $63 \%$ (HR $0.37 ; 95 \%$ confidence interval (CI) $0.25-0.54 ; \mathrm{p}<0.0001$ ) in the EURTAC study. One noteworthy finding of the phase III study is that, with an increase in median progression-free survival (PFS) from 5.2 to 9.7 months, some patients achieved a PFS of 3-4 years [6]. One important reason for this could be the influence of certain genetic changes on the prognosis and the efficacy of erlotinib.

\section{Non-ELREA Mutations}

While in the majority of patients with an activating EGFR mutation in exon 19 one of the usual E746_A750 deletions (ELREA mutations) is detected, a minority were found to have so-called non-ELREA mutations. With an $80 \%$ decrease in the risk of progression (HR 0.1957; 95\% CI 0.066$0.580 ; \mathrm{p}=0.0013)$, these patients in the EURTAC study benefited even more from the first-line therapy with erlotinib than the total population. While overall survival was significantly better in the erlotinib arm on detection of a nonELREA mutation than in patients with an ELREA mutation $(\mathrm{p}=0.0354)$, the difference in the chemotherapy arm was not statistically significant $(\mathrm{p}=0.650)(\mathrm{R}$. Rosell, unpublished data).

\section{Pre-Therapeutically Detected T790M Mutation}

In about one third of the patients with advanced NSCLC and proven activating EGFR mutation, the T790M mutation can additionally be detected before the treatment. Patients with pre-therapeutically detected T790M mutation had a significant advantage in the EURTAC study in both therapy arms in terms of PFS compared to patients without this mutation. The median total survival was also better in both therapy arms if the T790M mutation was also detected in addition to an activating EGFR mutation before the start of treatment (erlotinib arm: not reached vs. 16.1 months; chemotherapy arm: 22.6 months vs. 18.4 months) (R. Rosell, unpublished data). 


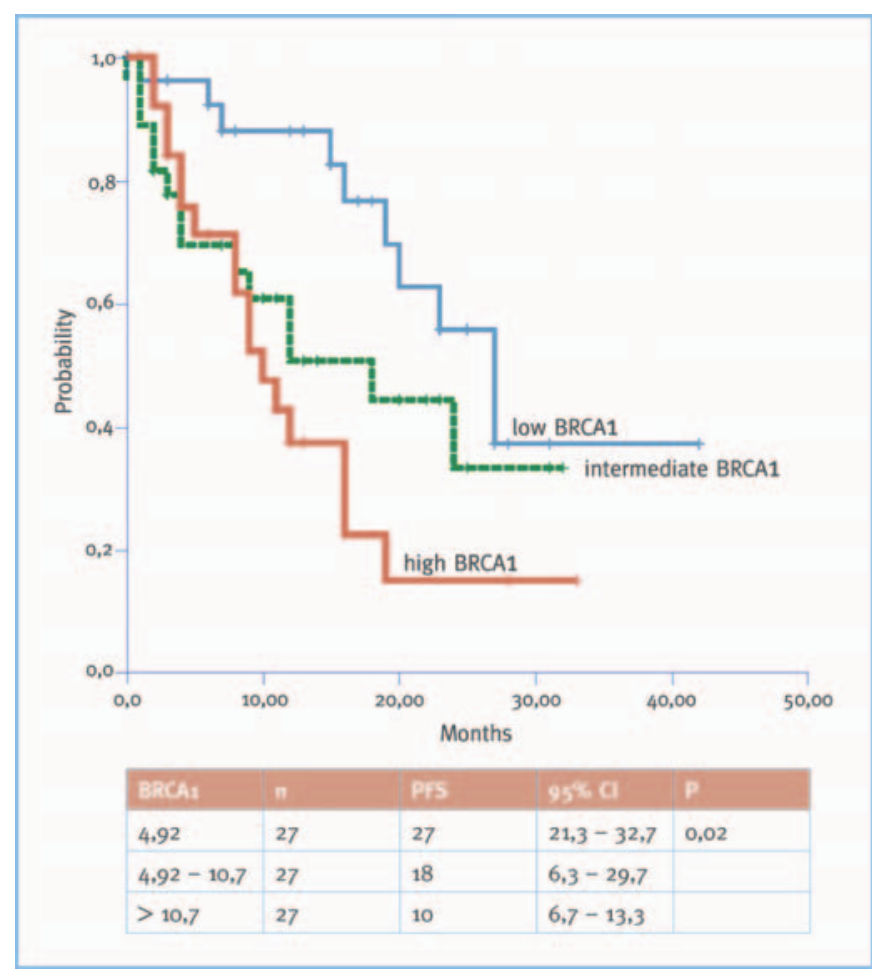

Abb. 2. Influence of BRCA1-mRNA level on PFS in erlotinib-treated patients with advanced NSCLC and proven activating EGFR mutation (modified according to [7]).

\section{Low BRCA1 Levels}

The expression of BRCA1 mRNA is also prognostically relevant in advanced NSCLC. In the screening study of the Spanish Lung Cancer Group, low BRCA1 mRNA levels were associated with much better PFS, independently of the presence of the T790M mutation (Abb. 2) [7].

This observation could also be therapeutically relevant because bevacizumab, through induction of tumor hypoxia, leads to the down-regulation of BRCA1; the addition of the VEGF antibody in patients with high BRCA1 mRNA levels could therefore represent an approach to improving the efficacy of erlotinib.

\section{Neue molekulare Targets - aktuelle Situation und Perspektive}

Molekulare Targets für eine personalisierte Therapie des NSCLC wurden zunächst bei Patienten mit Adenokarzinom identifiziert. Im Rahmen des globalen Clinical Lung Cancer Genome Project, das 2007 mit dem Ziel gestartet wurde, eine genomabhängige Taxonomie primärer Lungentumoren zu erstellen, wurden pathogenetisch relevante Veränderungen nun erstmals auch bei Patienten mit Plattenepithelkarzinom nachgewiesen.

In bis zu 20\% der Tumorproben fand die Lung Cancer Group Cologne häufige und fokale Amplifikationen des für einen Rezeptor des Fibroblasten-Wachstumsfaktors (fibroblast growth factor receptor1; FGFR1) codierenden Gens [8]. Nachdem experimentell eine Abhängigkeit FGFR1-amplifizierter Lungenkarzinom-Zelllinien von FGFR1 gezeigt werden konnte, wurde ein Fluoreszenz-in-situ-Hybridisierungs(FISH)-Assay zum Nachweis der FGFR1-Amplifikationen entwickelt. Die Wirksamkeit und Sicherheit des FGFR1Inhibitors BGJ398 bei entsprechend selektionierten Patienten werden in Köln zurzeit in einer Phase-I-Studie untersucht.

Bei etwa 3-4\% der Plattenepithelkarzinome sind aktivierende Mutationen des DDR2(discoidin domain receptor 2)Gens nachweisbar, die ebenfalls als onkogene Driver-Mutationen identifiziert wurden. Da im Maus-Xenograft-Modell des DDR2-mutierten Plattenepithelkarzinoms gezeigt werden konnte, dass das Tumorwachstum durch Dasatinib gehemmt wird, könnten von dem bereits bei Patienten mit PhiladelphiaChromosom-positiver chronischer und akuter myeloischer Leukämie (CML und AML) zugelassenen Kinase-Inhibitor zukünftig möglicherweise auch Patienten mit DDR2-mutiertem Plattenepithelkarzinom profitieren.

Insgesamt wurde bei etwa $45 \%$ der bei LungenkarzinomPatienten entnommenen Proben mindestens eine genetische Läsion entdeckt; für viele dieser onkogenen Veränderungen sind bereits therapeutische Ansätze verfügbar oder werden in präklinischen Modellen und klinischen Studien untersucht. Bei Patienten mit Plattenepithelkarzinom gelten neben FGFR1-Amplifikationen und DDR2-Mutationen vor allem Mutationen von FGFR2 und FGFR3 sowie PIK3CA-Mutationen als potenzielle Targets einer personalisierten Therapie. Patienten mit kleinzelligem Lungenkarzinom (SCLC) können bei Nachweis von FGFR1-Amplifikationen, die in 6\% der Tumorproben detektiert wurden, zukünftig möglicherweise ebenfalls von einer personalisierten Therapie profitieren.

Die Umsetzung der im Rahmen des Clinical Lung Cancer Genome Project gewonnenen Daten in Arzneimittel für genetisch selektionierte Subgruppen erfordert neben genauen Kenntnissen der Tumorbiologie und einer schnellen «realtime» genetischen Diagnostik eine intensive Zusammenarbeit von Grundlagenforschern, Pathologen und Klinikern, wie sie im Raum Köln/Bonn im Regional Network for Molecular Cancer Screening erfolgt. Bis Februar 2012 wurden hier mehr als 2400 Lungenkarzinom-Patienten auf pathogenetisch relevante molekulare Veränderungen untersucht und von der Lung Cancer Group Cologne mehrere Phase-I- und -II-Studien zur personalisierten Therapie des Lungenkarzinoms initiiert oder geplant.

\section{Entwicklungen in der molekulargenetischen Diagnostik des NSCLC}

Die Selektionierung der Patienten auf Basis der pathologisch relevanten genetischen Veränderungen erfordert eine leistungsstarke molekulare Diagnostik, wobei die Anforderun- 


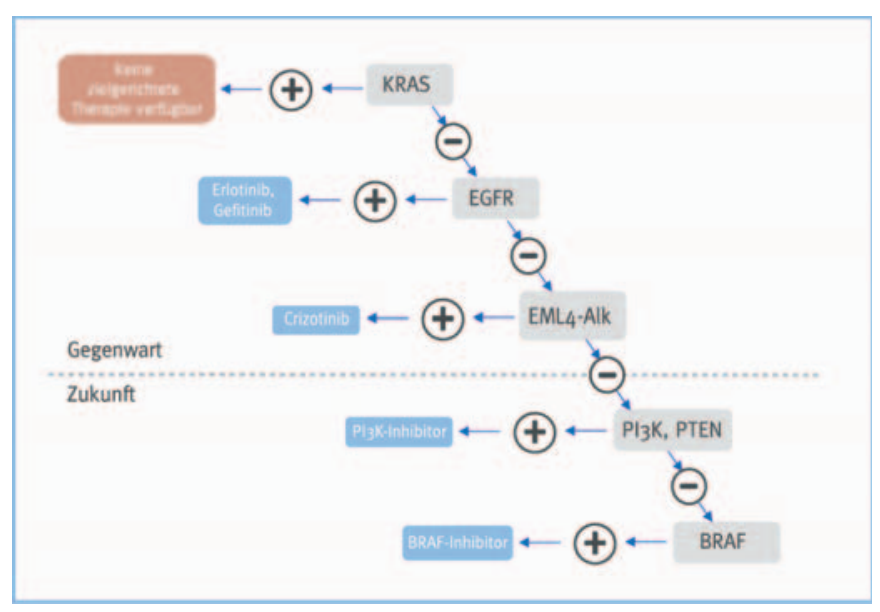

Abb. 3. Vorgeschlagener Algorithmus für die molekulargenetische Diagnostik des NSCLC.

gen mit der Identifikation weiterer therapierbarer molekularer Targets zukünftig noch zunehmen dürften. Eine zusätzliche Herausforderung ist dabei, dass bei Patienten mit inoperablem NSCLC für die molekulargenetische Diagnostik häufig nur Biopsien mit relativ wenigen Tumorzellen zur Verfügung stehen, die im Gewebe inhomogen verteilt sein können. Da gesicherte Aussagen zu genetischen Veränderungen bei der Sanger-Sequenzierung nur bei einem Tumorzellanteil von mindestens etwa $20 \%$ möglich sind, sollten bei kleinen Biopsien die Tumorzellen gegebenenfalls mithilfe der lasergesteuerten Mikrodissektion aus dem Gewebeschnitt separiert und angereichert werden.

\section{EGFR-Signalweg}

Die meisten der derzeit beim NSCLC für eine zielgerichtete Therapie relevanten Mutationen betreffen den EGFR mit der Signaltransduktion über KRAS, BRAF und MAP-Kinase (MAPK) oder PI3K, AKT und mTOR. Aktivierende Mutati- onen des EGFR werden bei europäischen Patienten mit fortgeschrittenem NSCLC in 10-15\% der Fälle nachgewiesen; betroffen sind überwiegend die Exons 19 (Deletionen) und 21 (Punktmutation L858R); EGFR-Mutationen können aber auch in den Exons 18 und 20 vorkommen, die daher ebenfalls in die Mutationsanalyse eingeschlossen werden sollten. Erworbene Resistenzen gegenüber EGFR-Tyrosinkinase-Inhibitoren (TKI) können auf die T790M-Mutation zurückgehen oder Folge eines onkogenen Kinase-Switch auf das cMETProtein sein.

Da sich die meisten der an der EGFR-vermittelten Signaltransduktion beteiligten Mutationen gegenseitig ausschließen, wurde unter Berücksichtigung der begrenzten Gewebeproben und ökonomischer Aspekte der in Abbildung 3 dargestellte Algorithmus vorgeschlagen. Bereits bei Berücksichtigung möglicher Veränderungen der KRAS-, EGFR-, ALK-, PI3K-, PTEN- und BRAF-Gene dürfte die sequenzielle Analyse einzelner Mutationen allerdings schon aus Zeitgründen an ihre Grenzen stoßen.

Erfolgversprechender ist die gleichzeitige Analyse aller infrage kommenden Mutationen bzw. die Erstellung eines individuellen genetischen Tumorprofils mittels Next Generation Sequencing (NSG). Die Pyrosequenzierung (454-Technologie) ermöglicht die gleichzeitige Sequenzierung von mehr als 100 Polymerase-Kettenreaktion (PCR)-amplifizierten Genabschnitten mehrerer Patienten, deren DNA zuvor mit genetischen Barcodes versehen wurde. Da beim NSG auch Tumoranteile von 1-2\% genau analysiert werden können, ist hier auch bei kleinen Biopsien mit inhomogener Verteilung der Tumorzellen keine Mikrodissektion erforderlich.

\section{Disclosure Statement}

Die Autoren waren Referenten beim «Dresdner Interdisziplinäres Gespräch 2012», das von Roche Pharma AG unterstützt wurde.

\section{Literatur}

1 Scagliotti G, Brodowicz T, Shepherd F, et al.: Pemetrexed is more effective in patients with nonsquamous non-small cell lung cancer (NSCLC) histology: an analysis of three large, randomized, phase III trials. J Thorac Oncol 2009;4(suppl 1): S325-S326.

2 Gandara DR, Grimminger PP, Mack PC, et al. Histology- and gender-related associations of ERCC1, RRM1, and TS biomarkers in 1802 patients with NSCLC: Implications for therapy. J Clin Oncol 2010;28(suppl 15s):abstr 7513.

3 Soria JC, Mauguen A, Reck M, et al.: Meta-analysis of randomized phase II/III trials adding bevacizumab to platin-based chemotherapy as 1 st-line treatment in patients with advanced non-small cell lung cancer (NSCLC). European Society for Medical Oncology (ESMO) Annual Meeting 2010; poster 437P.
4 Gandara DR, Mack PC, Li T, et al.: Evolving treatment algorithms for advanced non-small-cell lung cancer: 2009 looking toward 2012. Clin Lung Cancer 2009;10:392-394.

5 Cobo M, Isla D, Massuti B, et al.: Customizing cisplatin based on quantitative excision repair crosscomplementing 1 mRNA expression: a phase III trial in non-small-cell lung cancer. J Clin Oncol 2007;25:2747-2754.

6 Rosell R, Carcereny E, Gervais R, et al.: Erlotinib versus standard chemotherapy as first-line treatment for European patients with advanced EGFR mutation-positive non-small-cell lung cancer (EURTAC): a multicentre, open-label, randomised phase 3 trial. Lancet Oncol 2012;13:239-246.
7 Rosell R, Molina MA, Costa C, et al.: Pretreatment EGFR T790M mutation and BRCA1 mRNA expression in erlotinib-treated advanced non-smallcell lung cancer patients with EGFR mutations. Clin Cancer Res 2011;17:1160-1168.

8 Weiss J, Sos ML, Seidel D, et al.: Frequent and focal FGFR1 amplification associates with therapeutically tractable FGFR1 dependency in squamous cell lung cancer. Sci Transl Med 2010;2: 62ra93. 